\title{
Research Paper: Treatment of Distal End Clavicle Fractures: Suture Fixation Versus Tension Band Wiring
}

\author{
Mohsen Mardani-Kivi' ${ }^{1}$ (D, Mahmoud Karimi Mobarakeh² (iD, Ehsan Kazemnejad Leili ${ }^{\text {(D) }}$, Zahra Haghparast Ghadim-Limudahi ${ }^{* *}$ (D) \\ 1. Orthopaedic Research Center, Department of Orthopaedic, Poursina Hospital, School of medicine, Guilan University of Medical Sciences, Rasht, Iran \\ 2. Department of Orthopaedic, Faculty of Medicine, Kerman University of Medical Sciences, Kerman, Iran. \\ 3. Department of Statistics, School of Health, Guilan University of Medical Sciences, Rasht, Iran. \\ 4. Department of Orthopaedic, School of Medicine, Guilan University of Medical Sciences, Rasht, Iran.
}

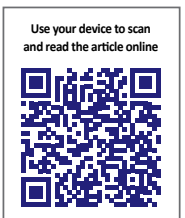

Citation Mardani-Kivi M, Karimi Mobarakeh M, Kazemnejad Leili E, Haghparast ghadim-limudahi Z. Treatment of Distal End Clavicle Fractures: Suture Fixation Versus Tension Band Wiring . Journal of Research in Orthopedic Science. 2021; 8(3):149-156. http://dx.doi.org/10.32598/JROSJ.8.3.772.1

hittp://dx.doi.org/10.32598/JROSJ.8.3.772.1

Keywords:

Clavicle, Fractures, Surgery

\begin{abstract}
A B S T RA C T
Background: Several methods like Kirschner wire, suture fixation, plaque, and so on are used for surgical treatment of Neer type-II fracture of the distal end of the clavicle.

Objectives: Because of no gold standard in treating fractures of the distal end of the clavicle, this study was conducted to compare therapeutic outcomes of Tension Band Wire (TBW) and Suture Fixation (SF) in treating this type of fracture.

Methods: This retrospective, analytical cross-sectional study was performed on patients with confirmed unilateral unstable Neer type-II fracture of the distal end of the clavicle. Based on the surgeon's preferred protocol for fixation of fractures, pin and wire were used for the patients operated from September 21, 2010, to March 20, 2013 (TBW group), and suture fixation for patients operated from March 21, 2013, to September 23, 2015, (SF group). Demographic information was recorded separately for both groups. All patients were evaluated and scored based on constant score, Visual Analog Scale (VAS), symptomatic hardware, and loss of reduction in 3 and 6 months, and final visit after surgery.

Results: Among 85 patients, 41 and 44 were allocated in TBW and SF groups, respectively. The mean follow-up time was 36.7 months. Our findings showed that both groups in three follow-up periods were similar in shoulder function based on constant score, VAS score, loss of reduction, and nonunion. But the SF group had lesser symptomatic hardware rather than the TBW group $(\mathrm{P}=0.001)$.

Conclusion: Although the measures of pain intensity and function were similar in the two groups, symptomatic hardware and the need for hardware removal in the TBW method are weak points that should be considered.
\end{abstract}

* Corresponding Author:

Zahra Haghparast Ghadim-Limudahi, MD.

Address: Department of Orthopaedic, School of Medicine, Guilan University of Medical Sciences, Rasht, Iran.

Phone: +98 (13) 33311470

E-mail: Dr.zahra.haghparast@gmail.com 


\section{Introduction}

he Neer type-II fracture of the distal end $\mathbf{T}$ of the clavicle is unstable in which acromioclavicular is intact, but the coracoclavicular ligament is torn [1]. Although this type of fracture accounts for a small percentage of all clavicular fractures (15-28\%), they are responsible for $30 \%-45 \%$ of nonunion cases [2]. Non-surgical methods were used to treat this type of fracture, but surgical techniques replaced them due to the high percentage of nonunion in distal fractures, accompanied by displacement [3].

There are several methods for surgical treatment of these fractures, such as fixation with Kirschner wire, suture fixation, different plaques, cerclage wire of broken parts, hook plate assisted osteosynthesis, Knowles pin, etc. [3-5]. Albeit, these methods are associated with some problems such as lack of fixation, screw protrusion, fracture, nonunion, malunion, and the potential need for hardware extraction [3].

A few high-quality research studies have been performed to find the best surgical method. In addition, there are no preferred methods for surgery of these types of fractures because of complications and the unstable nature of fractures of the distal end of the clavicle.

\section{Objectives}

Because of no gold standard in treating fractures of the distal end of the clavicle, we conducted to compare the therapeutic outcomes of Tension Band Wire (TBW) and Suture Fixation (SF) in treating this type of fracture.

\section{Methods}

\section{Study patients}

This retrospective, analytical cross-sectional study was approved by Institutional Review Board and performed on patients with confirmed unilateral unstable Neer typeII fracture of the distal end of the clavicle (rupture of the coracoclavicular ligament). Trauma to surgery duration was less than one week in all patients, and their shoulder function was normal. All surgeries were performed under general anesthesia and beach-chair position by a specialist shoulder surgeon (the first author). Patients from September 21, 2010, to March 20, 2013, were enrolled as the TBW group. Then with the possibility that SF might have better results, it was used for patients from March 21, 2013, to September 23, 2015.
The patients with a history of fracture of shoulder and clavicle, degenerative joint disease of acromioclavicular or shoulder joints, rheumatoid arthritis of acromioclavicular or shoulder joints were excluded. Also, heavy smokers, drug abusers, and those with psychological disorders were excluded from the study.

\section{Surgical methods}

In the TBW group, dissection was performed from the proximal end of the fracture site to the lateral in the event of the clavicle to the front of the acromion. The displaced clavicle was reduced to the proximal, and then two 2-mm pins were placed from acromion to clavicle from posterolateral to anteromedial direction. For better stability, the pins were fastened together with wire on the top of the clavicle and acromion to make tension band wiring fixation. The end of the pins was bent, and soft tissues and skin were sutured. In this method, hardware was extracted after 3 months if the union was completed (Figures 1 and 2). In the SF group, the Ethibond 5 suture was become two-layered and passed under the coracoid. Then two holes were created with $1 \mathrm{~cm}$ distance from superior to inferior in the clavicle and after open fracture replacement. Each fiber of Ethibond 5 suture was passed through holes from inferior to superior of the clavicle and finally were sutured and tightened with each other.

\section{Postoperation follow-up}

Rehabilitation in the two groups was similar. All shoulders were placed in a sling for 3 weeks. Active movements of the elbow, wrist, and hand began one day after the operation. Passive and active assisted exercises began 3 weeks after the operation, and shoulder rehabilitation after 6 weeks. Heavy exercise or practice was allowed 3 months after the operation.

Sutures were removed 2 weeks after the operation. The shoulder x-ray was performed every 2 weeks for 3 months to evaluate the fusion status. Passing osseous trabeculae through fracture site in at least 3 of 4 cortexes in radiographic images was considered a union. If the union did not occur after 3 months, it was considered nonunion.

\section{Study measurements}

All patients were evaluated and scored based on Constant Scores (CS) (a 100-point common scoring system about shoulder function status, including pain, daily functions, movement amplitude, and joint power). The difference in constant score was measured on both sides, 


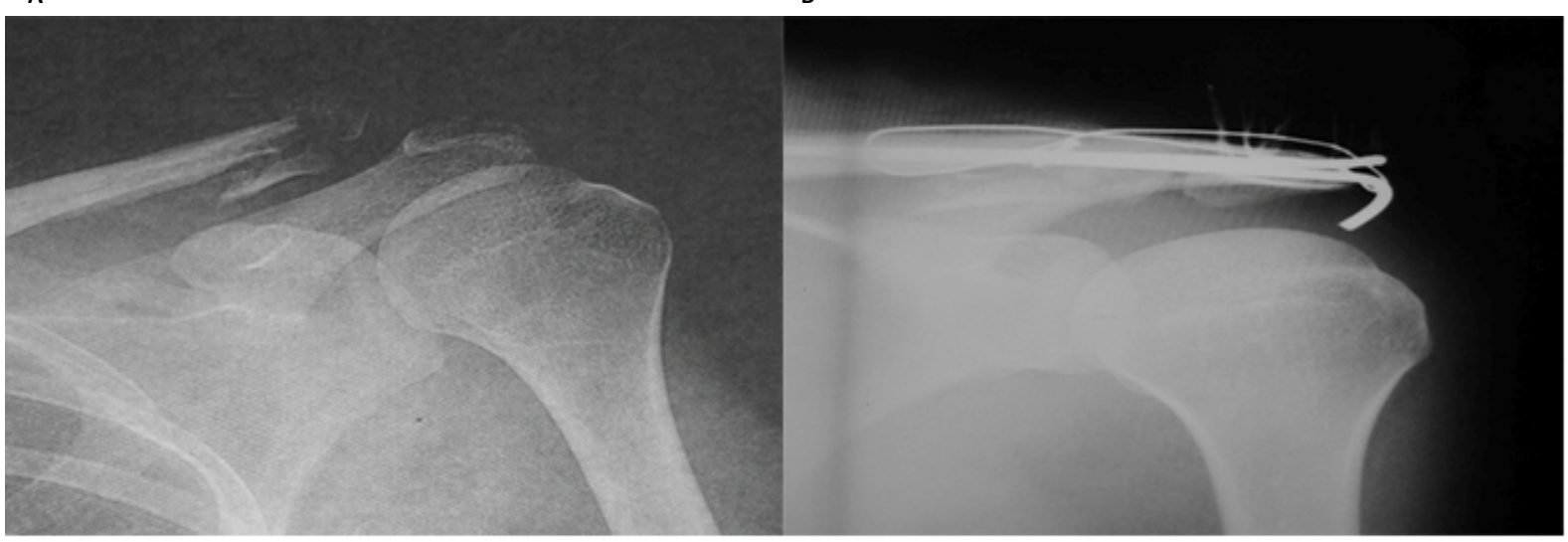

Figure 1. (A) A 27-year-old male with fracture of the left distal clavicle; (B) Treated with tension band wire

Orthopedic Science and according to Fabre classification, it has four groups: excellent (difference $<11$ ), good (11-20), (moderate (21$30)$, and poor $(>30)$ [6]. The pain was evaluated by Visual Analog Scale (VAS) from 0-10 scores in three positions of resting $(<3.5 \mathrm{kcal} / \mathrm{min})$, moderate $(3.5-7 \mathrm{kcal} /$ $\mathrm{min}$ ), and severe ( $>7 \mathrm{kcal} / \mathrm{min}$ ) activity $[7,8]$. Symptomatic hardware included any discomfort, leakage, or pain in the pin location, Loss of Reduction (LOR) which was determined as more than $50 \%$ increase in coracoclavicular space than the other side. Follow-up was performed at 3 and 6 months and final visit. Basic information such as age and gender were recorded from patients' files separately for both TBW and SF groups.

\section{Statistical analysis}

In this study, the independent $\mathrm{t}$ test was done to compare quantitative variables such as VAS and CS scores between the two groups at each follow-up session. The significance level was considered $\mathrm{P}<0.05$. All data were analyzed in SPSS version 19.

\section{Results}

Among 85 patients, 59 (69.41\%) were men, and 26 $(35.59 \%)$ were women. TBW group comprised 41 patients (25 men and 16 women) with a mean \pm SD age of $42.68 \pm 11.45$ years, and the SF group comprised 44 patients (34 men and 10 women) with a mean \pm SD age of $41.16 \pm 11.46$ years. Both groups were matched in terms of age and sex. The mean follow-up time was 36.7 months (range: 24-49 months). In the 6-month followup, 1 and 2 patients and at the final follow-up, 3 and 5 patients were excluded from TBW and SF groups, respectively (because they were unavailable). Our findings demonstrated that both groups in all follow-up periods were similar with regard to shoulder function based on CS (Table 1, P $>0.05$ ).

Comparison of patients' pain based on VAS score in three conditions of rest, moderate, and strenuous activi-
A

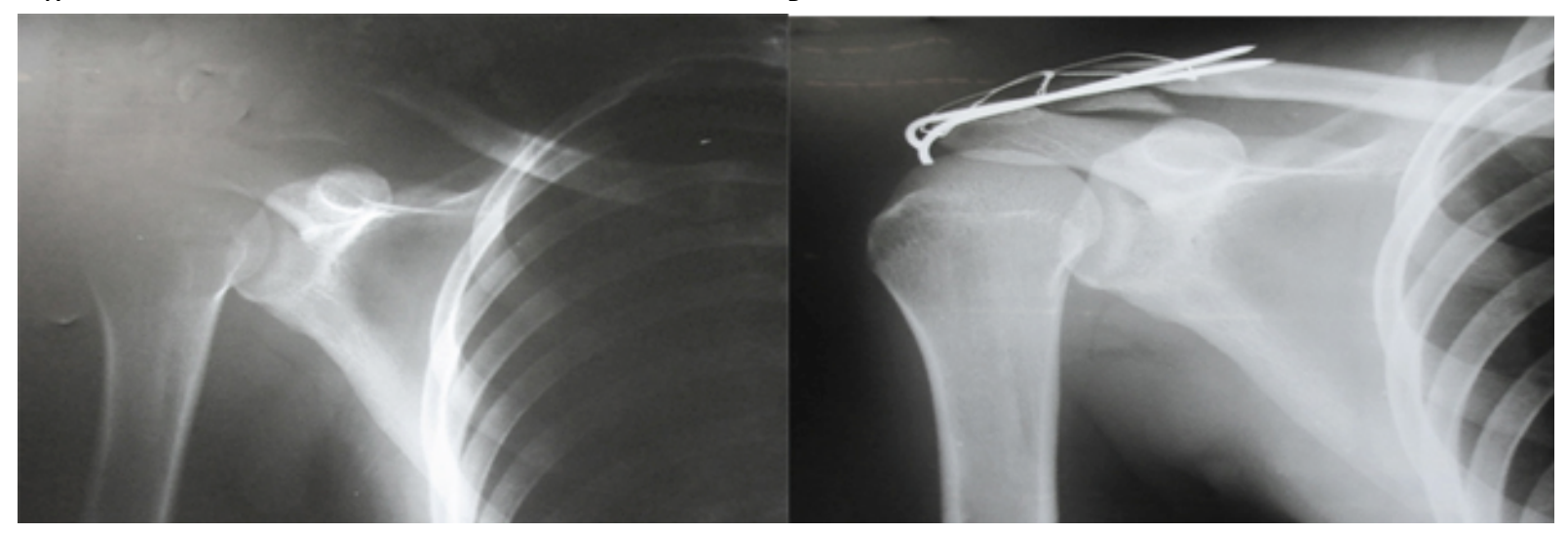

Orthopedic Science

Figure 2. (A) Right distal clavicle fracture in a 33-year-old male; (B) After tension band wiring 


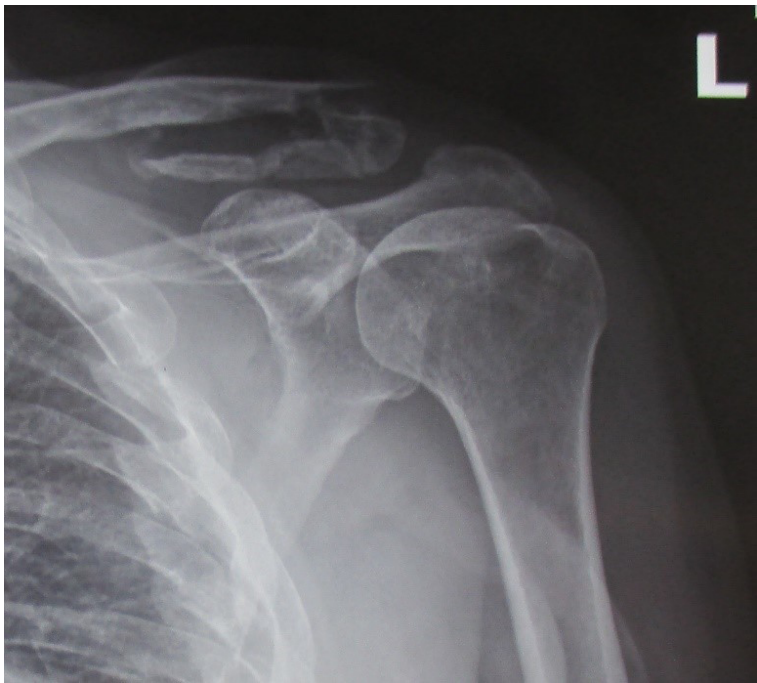

Orthopedic Science

Figure 3. Nonunion after removal of the pins in a 38-year-old male

ties are presented in Table 2. Our findings showed that pain in both groups was similar in all follow-up points. Pain intensity after moderate and severe activities in 3-month follow-up showed a considerable difference between the two groups and was higher in the TBW group. However, this difference was not statistically significant.

Symptomatic hardware was not seen in the SF group because no pin was used in this group. In the first 3-month follow-up of the TBW group, 11 patients $(28.2 \%)$ had a superficial infection in pin location, which was recovered completely after pin extraction. Deep infection and wound discharge were not seen in both groups. No patients in the TBW group and 2 patients in the SF group had LOR; this difference was not statistically significant $(\mathrm{P}=0.692)$. Our findings showed that 2 patients from the TBW group and 3 patients from the SF group had nonunion $(\mathrm{P}=0.308)$ (Figure 3$)$.

\section{Discussion}

One of the most common causes of referral to orthopedics is clavicle fractures. These fractures account for up to $44 \%$ of all shoulder girdle fractures in adults [9]. There are several surgical procedures for Neer type II distal end of the clavicle fractures, all of which are acceptable to treat this type of fracture. But on the other hand, there are many reasons to reject any of these methods as the gold standard for treating distal clavicle fractures $[10,11]$. Furthermore, Neer type II fractures are the causes of $67 \%-75 \%$ of nonunion or delayed unions [2], so finding the proper surgical technique to treat this type of fracture is essential.

The results of TBW and SF groups were similar regarding pain intensity, functional outcomes (constant score), LOR, and nonunion rates. Still, few differences between them were not statistically significant. Patients in the TBW group showed better functional, LOR, and nonunion outcomes. Park et al. showed in their study

Table 1. Functional outcome

\begin{tabular}{|c|c|c|c|c|}
\hline \multirow{2}{*}{\multicolumn{2}{|c|}{ Follow-Up }} & \multicolumn{2}{|c|}{ Groups, No. } & \multirow{2}{*}{$\mathbf{P}$} \\
\hline & & Tension Band Wire & Suture Fixation & \\
\hline \multirow{5}{*}{ Three months } & Excellent & 25 & 30 & \multirow{5}{*}{0.339} \\
\hline & Good & 13 & 12 & \\
\hline & & & & \\
\hline & Moderate & 2 & 1 & \\
\hline & Poor & 1 & 1 & \\
\hline \multirow{5}{*}{ Six months } & Excellent & 32 & 36 & \multirow{5}{*}{0.429} \\
\hline & Good & 6 & 6 & \\
\hline & & & & \\
\hline & Moderate & 0 & 1 & \\
\hline & Poor & 1 & 0 & \\
\hline \multirow{5}{*}{ Final } & Excellent & 35 & 37 & \multirow{5}{*}{0.461} \\
\hline & Good & 1 & 1 & \\
\hline & & & & \\
\hline & Moderate & 0 & 0 & \\
\hline & Poor & 0 & 0 & \\
\hline
\end{tabular}


Table 2. Comparing pain intensity based on visual analog scale between the two groups in three different follow-up times

\begin{tabular}{|c|c|c|c|c|}
\hline \multirow{2}{*}{ Conditions } & \multirow{2}{*}{ Follow-up Time } & \multicolumn{2}{|c|}{ Mean \pm SD } & \multirow{2}{*}{$\mathbf{P}$} \\
\hline & & Tension Band Wire Group & Suture Fixation Group & \\
\hline \multirow{3}{*}{ Rest } & Three months & $1.16 \pm 0.987$ & $0.974 \pm 0.93$ & \multirow{3}{*}{0.352} \\
\hline & Six months & $0.963 \pm 0.95$ & $0.905 \pm 0.86$ & \\
\hline & Final & $0.622 \pm 0.41$ & $0.462 \pm 0.20$ & \\
\hline \multirow{3}{*}{ Moderate activity } & Three months & $2.34 \pm 0.963$ & $2.05 \pm 0.914$ & \multirow{3}{*}{0.238} \\
\hline & Six months & $1.43 \pm 0.900$ & $1.23 \pm 0.937$ & \\
\hline & Final & $0.625 \pm 0.57$ & $0.532 \pm 0.36$ & \\
\hline \multirow{3}{*}{ Severe activity } & Three months & $4.02 \pm 1.229$ & $3.57 \pm 1.784$ & \multirow{3}{*}{0.410} \\
\hline & Six months & $1.64 \pm 0.750$ & $1.57 \pm 0.661$ & \\
\hline & Final & $0.75 \pm 0.686$ & $0.55 \pm 0.548$ & \\
\hline
\end{tabular}

Orthopedic Science

that TBW is an effective method and has an acceptable outcome [12]. These better results may be due to more rigid fixation, which was applied in this group. However, the pain intensity in 3 months follow-up was higher in the TBW group compared to the SF group. As said in the surgical technique, subcutaneous pins were used for fixation in the TBW group for the first 3 months after surgery, and pins may cause more pain in the patients of this group at 3 months follow-up.

The main significant difference between the two groups was the symptomatic hardware rate. This means that in the first follow-up time, $28.2 \%$ of patients in the TBW group had superficial infection, but this complication was not seen in the other group. The occurrence of symptomatic hardware is one of the main disadvantages in the TBW group, which is due to the used pins. It is possible that if we placed the pins more deeply, the rate of symptomatic hardware and subsequent pain would reduce. Another issue is the extra surgery to extract the pins. In general, surgical procedures are beneficial, but they may have potential adverse effects. Patients are at risk of post-operation complications since they receive anesthetics. So it is clear that with a higher number of surgeries, the possibility of such dangers and complications would increase. Therefore, techniques in which patients undergo a single surgery are safer than those that need further operations.

Another important topic in selecting appropriate surgical methods is the costs of the two methods. Although in the study, the cost of each method was not evalu- ated precisely, it seems that the SF method is the more cost-effective because of the single surgery. However, in the TBW method, the patients may undergo surgery one more time: first for the fixation of the fracture and next for hardware removal. This second surgery forced patients to have a second hospitalization and a second absence from working. This absence may lead to lower income for them. So, this situation by itself imposes more expenses on patients. This higher cost is not just related to the patients but also to the health care system, which will experience considerable expenses for the two surgeries with respect to the workforce, time, and equipment compared to a single surgery. It seems that the SF method in which the patient undergoes a single surgery without postoperative complications is a more suitable choice.

In the study by Tseui et al., two surgical methods of TBW and Transacromial Kitschner Wire (TKW) were compared to treat acute unstable distal clavicle fracture [2]. In their study, the TBW group was significantly better than the TKW group with regard to shoulder function and occurrence of complications $(\mathrm{P}=0.021$ and $\mathrm{P}=0.005$, respectively). Although in their study, similar to our study, severe complications such as deep infection or pin migration, which resulted in organ injuries, were not seen in any of the groups: $43 \%$ of TKW patients and $7 \%$ of TBW patients suffered from pin tract infection. They extracted the pins of TKW three weeks after the operation and three months after surgery in TBW groups to prevent more infection and patient discomfort. Union happened earlier and in all patients in TBW compared to TKW, but this time difference was not significant. 
However, the study of Tseui et al. [2] had one important problem, which the authors also stated. They had a low number of studied patients (29 cases), and this low sample size decreases the reliability of their findings. Unlike them, we had a larger sample size to increase the reliability of our findings.

Yih-shiunn Lee et al. compared two methods of Hook Plate (HP) and TBW in 52 patients with unstable distal clavicle fractures [13]. In their study, patients of the HP group had better status regarding union, shoulder condition, return to activity, and symptomatic hardware than the TBW group. In the HP group, one patient (3.1\%) was complicated by partial LOR. But in the TBW group, 6 patients had complications: 3 partial LOR, 1 complete LOR with nonunion, and 2 superficial infections. These two cases of infections were diagnosed in the first postoperation follow-up and treated with antibiotics. Another important issue in their study is that $85 \%$ of patients in the TBW group suffered symptomatic hardware, while $34.3 \%$ of HP patients suffered from this complication, and this difference was statistically significant $(\mathrm{P}=0.001)$. Finally, they recommended two surgical methods for treating this type of fracture but stated that HP is preferred to TBW due to better progress and fewer complications. One of the problems of their study, which authors also expressed, was that seven different resident orthopedists performed the surgeries. Therefore, differences in the experience and skill levels of residents might have affected the study's outcomes. We overcame this problem by having all surgeries performed by one surgeon (first author).

In addition to the lack of surgical cost calculation, the retrospective nature of the study and performing without randomization are also the limitations of this study that must be considered in future studies.

\section{Conclusion}

Although the outcomes of pain intensity and function were similar in the two groups, symptomatic hardware and the need for hardware removal in the TBW method are weak points that should be considered.

\section{Ethical Considerations}

\section{Compliance with ethical guidelines}

The project was approved by Islamic Azad University, Tonekabon Branch, Iran.

\section{Funding}

This research did not receive any grant from funding agencies in the public, commercial, or non-profit sectors.

\section{Authors' contributions}

Study concept and design, and study supervision: Mohsen Mardani-Kivi; Investigation: Mohsen MardaniKivi and Mahmoud Karimi Mobarakeh; Analysis and interpretation of data: Zahra Haghparast ghadim-limudahi; Drafting of the manuscript: Mohsen Mardani-Kivi and Zahra Haghparast ghadim-limudahi; Critical revision of the manuscript for Important intellectual content: Mohsen Mardani-Kivi and Mahmoud Karimi Mobarakeh; Statistical analysis: Zahra Haghparast ghadim-limudahi and Ehsan Kazemnejad Leili.

\section{Conflict of interest}

The authors declared no conflict of interest.

\section{Acknowledgments}

We thank Dr Khashayar Saheb Ekhtiari for his insightful suggestions.

\section{References}

[1] Stegeman SA, Nacak H, Huvenaars KHJ, Stijnen T, Krijnen P, Schipper IB. Surgical treatment of Neer type-II fractures of the distal clavicle: A meta-analysis. Acta Orthop. 2013; 84(2):18490. [DOI:10.3109/17453674.2013.786637] [PMID] [PMCID]

[2] Tsuei YC, Au MK, Chu W. Comparison of clinical results of surgical treatment for unstable distal clavicle fractures by transacromial pins with and without tension band wire. J Chin Med Assoc. 2010; 73(12):638-43. [DOI:10.1016/S17264901(10)70139-8] [PMID]

[3] Andersen JR, Willis MP, Nelson R, Mighell MA. Precontoured superior locked plating of distal clavicle fractures: A new strategy. Clin Orthop Relat Res. 2011; 469(12):3344-50. [DOI:10.1007/s11999-011-2009-5] [PMID] [PMCID]

[4] Arciero RA. Operative techniques for displaced distal clavicle fractures. Oper Tech Sports Med. 2004; 12(1):27-31. [DOI:10.1053/j.otsm.2004.04.005]

[5] Klein SM, Badman BL, Keating CJ, Devinney DS, Frankle MA, Mighell MA. Results of surgical treatment for unstable distal clavicular fractures. J Shoulder Elbow Surg. 2010; 19(7):1049-55. [DOI:10.1016/j.jse.2009.11.056] [PMID]

[6] Fabre T, Piton C, Leclouerec G, Gervais-Delion F, Durandeau A. Entrapment of the suprascapular nerve. J Bone Joint Surg Br. 1999; 81(3):414-9. [DOI:10.1302/0301620X.81B3.0810414] [PMID] 
[7] Ainsworth BE, Haskell WL, Leon AS, Jacobs Jr DR, Montoye HJ, Sallis JF, et al. Compendium of physical activities: Classification of energy costs of human physical activities. Med Sci Sports Exerc. 1993; 25(1):71-80. [DOI:10.1249/00005768199301000-00011] [PMID]

[8] Centers for Disease Control and Prevention (CDC), National Center for Chronic Disease Prevention and Health Promotion (NCCDPHP), Division of Nutrition and Physical Activity (DNPA). Promoting physical activity: A guide for community action. Champaign: Human Kinetics; 1999. https:/ / books.google.com/books?id=bVrcwAEACAAJ\&dq

[9] Nordqvist A, Petersson C. The incidence of fractures of the clavicle. Clin Orthop Relat Res. 1994; 300:127-32. [DOI:10.1097/00003086-199403000-00016]

[10] Sambandam B, Gupta R, Kumar S, Maini L. Fracture of distal end clavicle: A review. J Clin Orthop Trauma. 2014; 5(2):65-73. [DOI:10.1016/j.jcot.2014.05.007] [PMID] [PMCID]

[11] Oh JH, Kim SH, Lee JH, Shin SH, Gong HS. Treatment of distal clavicle fracture: A systematic review of treatment modalities in 425 fractures. Arch Orthop Trauma Surg. 2011; 131(4):525-33. [DOI:10.1007/s00402-010-1196-y] [PMID]

[12] Park JY, Seo JB, Kim MH, Yu JW. [Tension band fixation for type II fracture of the distal clavicle (Korean)]. J Korean Fract Soc. 2005; 18(4):421-5. [DOI:10.12671/jkfs.2005.18.4.421]

[13] Lee YS, Lau MJ, Tseng YC, Chen WC, Kao HY, Wei JD. Comparison of the efficacy of hook plate versus tension band wire in the treatment of unstable fractures of the distal clavicle. Int Orthop. 2009; 33(5):1401-5. [DOI:10.1007/ s00264-008-0696-7] [PMID] [PMCID] 
This Page Intentionally Left Blank 